\title{
APPROXIMATE DESCRIPTION OF MULTI-SOLUTE ADSORPTION RATE IN ORGANIC AQUEOUS SOLUTION
}

\author{
Hiroyuki KAGE, IkUya HASHIZUME, Morio OKAZAKI ANd Ryozo TOEI \\ Department of Chemical Engineering, Kyoto University, Kyoto 606
}

\begin{abstract}
Wastewater generally contains many kinds of pollutants, and in most cases the composition of the pollutants is unknown. Most studies proposed in the past for liquid-phase adsorption, however, deal with solutions whose compositions are known, so it is not easy to apply the proposed methods to wastewaters. The authors proposed an approximate description for adsorption equilibrium by introduction of a new concept, "Characteristic Distribution of Langmuir Coefficient for Liquid Concentration (C.D.C.)"4). By using this description, it becomes possible to express the adsorption equilibrium in terms of total concentration index of solutes, for example TOC, in wastewater.

The object of the present study is to propose an approximate description of the adsorption rate characteristic of wastewater for which solutes and composition of the pollutants are unknown, and the concept of C.D.C. is applied to the adsorption rate of wastewater. These descriptions of adsorption rate and equilibrium of wastewater may be used in the design of adsorption columns for the treatment of wastewater.
\end{abstract}

\section{Theory}

Here let us consider the rate of concentration change of wastewater for which C.D.C. is known, in a batch adsorption operation. The C.D.C. of arbitrary wastewater can be obtained by Method I, which shows the most reliable results among the three methods proposed in the previous paper ${ }^{4}$. Next, the following assumptions are made.

1. Adsorbent particles are spherical.

2. Mass transfer resistance in liquid film is negligible.

3. Adsorption rate obeys the linear driving force approximation (L.D.F.A.), where driving force is due to the gradient of solid-phase concentration.

For the single-solute adsorption rate, more exact methods, in which the distribution of solid-phase concentration is considered, have been proposed $^{2,5}$. In

Received June 4, 1980. Correspondence concerning this article should be addressed to M. Okazaki. H. Kage is now with Kyushu Inst. of Tech., Kitakyushu 804. this paper, however, the L.D.F.A., proposed by Glückauf $^{11}$ and recently used for the analysis of adsorption columns ${ }^{31}$, is adopted in spite of its rough approximation. This is because the object of this paper is to describe the rate of adsorption of unknown multi-components in wastewater and so it is difficult to apply the more exact method to this study. Assumptions 1 and 2 agree with the experimental conditions mentioned in the section, "Experiments".

In the previous paper", the authors proposed the concept of C.D.C. to describe the adsorption equilibrium of a multi-solute system, and the purpose of this paper is to extend the concept of C.D.C. to the adsorption rate of the multi-solute system. When L.D.F.A. is adopted, the adsorption rate equation is represented by the product of $k_{s} a$ and the driving force. Since the driving force is represented by C.D.C. of the original solution, $k_{s} a$ can be assumed to be a function of $x$. Therefore this function, $k_{s}(x) a$, is dependent on adsorbates and adsorbent.

From the above descriptions, the following rate equation is obtained.

$$
d q(x, t) / d t=k_{s}(x) a\left\{q^{*}(x, t)-q(x, t)\right\}
$$

As the volume of solution in adsorbent can be neglected, from the material balance of the batch adsorption process,

$$
C(x, 0)-C(x, t)=A q(x, t)
$$

where $A$ is amount of adsorbent per unit volume of solution. When multi-solute adsorption equilibrium is assumed to obey the Markham-Benton equation ${ }^{4}$, Eq. (3) can be obtained from Eqs. (1) and (2).

$$
\begin{aligned}
d q(x, t) / d t= & k_{s}(x) a[Q(x) x\{C(x, 0)-A q(x, t)\} / \\
& \left.\left\{1+\int_{0}^{\infty}(C(x, 0)-A q(x, t)) x d x\right\}-q(x, t)\right]
\end{aligned}
$$

where $Q(x)$ is a function defined in Eq. (11) in the previous paper ${ }^{4)}$, and $C(x, 0)$ and $Q(x)$ can be obtained beforehand from the observed differential adsorption equilibrium curves (D.A.E. curves) by Method I of the previous paper. And Eq. (4) is the initial condition of Eq. (3).

$$
q(x, 0)=0
$$

From Eq. (2) the total concentration, $C_{T}(t)$, is 


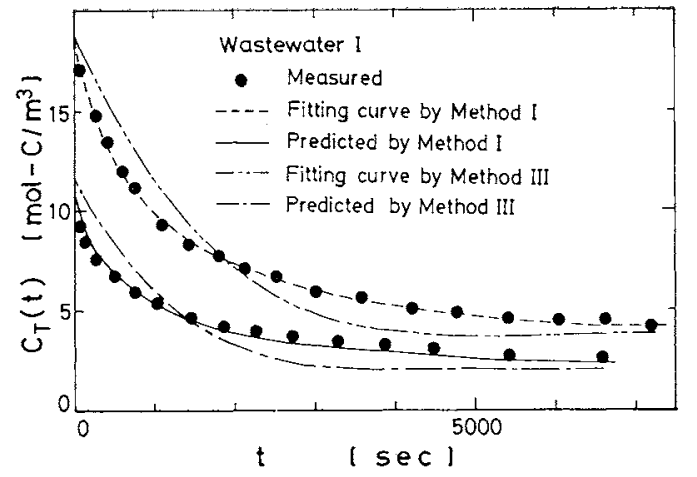

(adsorbent: activated carbon $\mathrm{X}-7000,35^{\circ} \mathrm{C}$ ) $\beta_{0}=1.90 \times 10^{-6} \mathrm{~s}^{-1}, \quad \beta_{1}=4.45 \times 10^{-4} \mathrm{~mol}-\mathrm{C} /$ $\mathrm{s} \cdot \mathrm{m}^{3}, \quad \beta_{2}=2.00 \times 10^{-3}(\mathrm{~mol}-\mathrm{C})^{2} / \mathrm{s} \cdot \mathrm{m}^{6}$

Fig. 1 Rate curves of concentration change

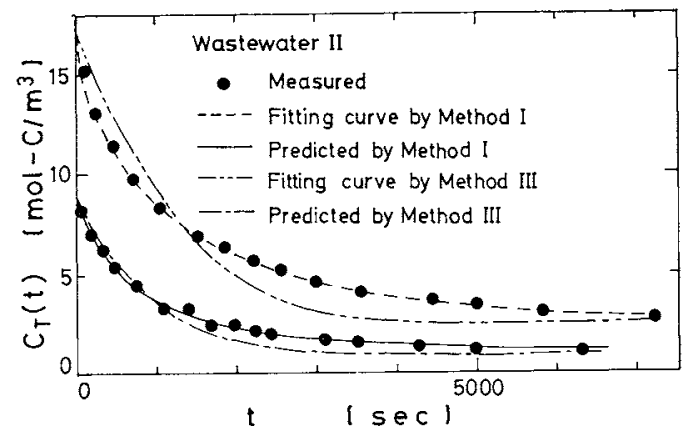

(adsorbent: activated carbon $X-7000,35^{\circ} \mathrm{C}$ ) $\beta_{0}=6.34 \times 10^{-5} \mathrm{~s}^{-1}, \quad \beta_{1}=1.42 \times 10^{-4} \mathrm{~mol}-\mathrm{Cl}$ $\mathrm{s} \cdot \mathrm{m}^{3}, \quad \beta_{2}=1.82 \times 10^{-3}(\mathrm{~mol}-\mathrm{C})^{2} / \mathrm{s} \cdot \mathrm{m}^{6}$

Fig. 2 Rate curves of concentration change

$$
C_{T}(t)=\int_{0}^{\infty} C(x, t) d x=C_{T}(0)-A \int_{0}^{\infty} q(x, t) d x
$$

In the practical calculation for the determination of $k_{s}(x) a$, we adopted Eq. (6) as a simulation formula.

$$
k_{s}(x) a=\beta_{0}+\beta_{1} x+\beta_{2} x^{2}
$$

In this case, the parameters to be searched are three, that is, $\beta_{0}, \beta_{1}$ and $\beta_{2}$. The practical calculation for determination of the parameters was performed as follows. As $C(x, 0), Q(x)$ and $A$ are known, when $\beta_{0}, \beta_{1}$ and $\beta_{2}$ in Eq. (6) are assumed arbitrarily, Eq. (3) can be solved with Eqs. (4) and (6) by the RKG method. The $q(x, t)$ obtained is substituted into Eq. (5), and $C_{T}(t)$ is obtained for the assumed parameters. Therefore, the optimal values of the parameters are searched so as to fit $C_{T}(t)$ to the observed curve by the SIMPLEX method. On the other hand, the prediction of $C_{T}(t)$ for the system of known parameters can be performed by solving Eq. (3) with Eqs. (4) and (6) and by substituting $q(x, t)$ into Eq. (5).

\section{Experiment}

To verify the applicability of the present method, it is required to show that $k_{s}(x) a$ determined for a wastewater can be applied to other wastewaters which
Table 1 Errors of each method

\begin{tabular}{ccccc} 
& \multicolumn{2}{c}{ Wastewater I } & \multicolumn{2}{c}{ Wastewater II } \\
Method & I & III & I & III \\
\hline$F_{1}^{*}$ & 0.0273 & 0.1340 & 0.0380 & 0.1508 \\
$F_{2}^{*}$ & 0.1023 & 0.2431 & 0.0647 & 0.1714 \\
\hline
\end{tabular}

$F_{1}$ : Fitting error of curve of concentration change (for upper curves in figures)

$F_{2}$ : Prediction error of curve of concentration change (for lower curves in figures)

* $F=(1 / N) \sum_{i}^{N}\left|C_{T i \text { eale }}-C_{T i \text { exp }}\right| / C_{T i \text { exp }}$,

$N$ : No. of experimental points

have the same set of contaminants as does the former, but in different concentrations. Then, the following experimental scheme was adopted. 1. A rate curve of concentration change for a wastewater was observed by using TOC analyzer, and $k_{s}(x) a$ was determined from it. 2. Another curve for a wastewater diluted by adding some amount of pure water was observed, and was compared with the predicted curve by using the $k_{s}(x) a$ determined for the former wastewater.

The experiments were performed using two kinds of synthetic wastewaters. They were aqueous solutions which respectively solved cyclohexanol, phenol, benzene, aniline, $p$-cresol and $p$-nitrophenol $(0.167$ : $0.167: 0.166: 0.167: 0.166: 0.167$, mol fraction based on mol-C) (Wastewater I), and $p$-chlorophenol, $p$ -

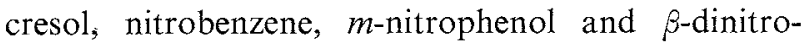
phenol (0.141:0.282:0.211:0.253:0.113) (Wastewater II). Spherical activated carbon from Takeda Chemical Industry Co., Ltd., X-7000 $(0.154 \mathrm{~cm}$ average diameter), was used as adsorbent. The procedure of the differential adsorption experiment for the determination of C.D.C. was exactly the same as that described in the previous paper ${ }^{4}$. The experiment for adsorption rate was performed by the usual batch method. As the speed of the impeller was sufficiently fast, the mass transfer resistance in the liquid film was negligible.

\section{Results and Discussion}

The experimental results for adsorption rate are shown in Figs. 1 and 2 . Using the parameters obtained from D.A.E. curves, $k_{s}(x) a$ was determined from the curve of concentration change which has the higher initial concentration of the two curves for each wastewater, and $C_{T}(t)$ curves calculated by the uses of the determined $k_{s}(x) a$ (fitting curves) are plotted by a broken line in Figs. 1 and 2. Further, the values obtained of $\beta_{0}, \beta_{1}$ and $\beta_{2}$ in Eq. (6) and the errors are shown in Figs. 1 and 2 and also Table 1. As shown in Figs. 1 and 2, the agreement between the fitting curves and the measured ones is so good that the simulation formula, Eq. (6), can be considered sufficient for the representation of $k_{s}(x) a$. The predicted 
rate curves for lower initial concentrations are shown by a solid line in Figs. 1 and 2, by the use of Eq. (3) and $k_{s}(x) a$ obtained above, and are compared with the experimental results. The prediction errors are shown in Table 1. Let us call this description technique Method I in this paper.

Method $\mathrm{I}$ is compared with the lumped parameter method, Method III, to verify its usefulness. In Method III, the solutes in wastewaters are considered to consist of one unadsorbable and one adsorbable solute, which obeys the Langmuir equation. This is the same treatment of Method III as in the previous paper $^{4}$. The L.D.F.A. is adopted for the adsorption rate, and the fitting curves and curves predicted by Method III are also shown in Figs. 1 and 2. The fitting and prediction errors are shown in Table 1 for comparison with Method I. It is clear from Figs. 1 and 2 or Table 1 that Method III is inferior to Method I.

Next let us compare the values obtained of $k_{s}(x) a$ with the proposed experimental data of Suzuki et $a l .^{6}$, which were measured for various single organic solutes. The component corresponding to the value of $x$, which gives the maximum value of $C$ of C.D.C. for each wastewater, is regarded as the most influential solute in the adsorption system, and $k_{s}(x) a$ for this value of $x$ is calculated. The value of $k_{s}(x) a$ is further converted into effective surface diffusion coefficient, $D_{s}$, by the relationship $k_{s} a \simeq 15 D_{s} / R^{21}$, and we can get $D_{s} \simeq 4.51 \times 10^{-8} \mathrm{~cm}^{2} / \mathrm{s}$ for Wastewater I and $4.70 \times$ $10^{-8}$ for Wastewater II. These values of $D_{s}$ lie in the same order of magnitude as the experimental data of Suzuki et $\left.a l .{ }^{6}\right)$ for the respective solute. By considering the difference in experimental conditions, the $k_{s}(x) a$ obtained seems to be reliable.

For aqueous solutions which contain many unknown organic solutes, the method proposed here (Method I) will be useful for estimation of the adsorption rate, though the rough approximation of driving force is employed.

\section{Acknowledgment}

The authors are grateful to Messrs. R. Nagashima, J. Hirano and T. Takahashi for their assistance in the experimental work, to the Ministry of Education, Science and Culture, Japan for the awards of Grant-in-Aid for Scientific Research, Special Project Research, No. 011917 (1975), 111213 (1976) and 210416 (1977), and to Takeda Chemical Industry Co., Ltd. for supplying activated carbon.

\section{Nomenclature}

$A \quad=$ amount of adsorbent used $\quad\left[\mathrm{kg} / \mathrm{m}^{3}\right]$

$\mathrm{C}=$ probability density of liquid-phase TOC

concentration $\left[(\mathrm{mol}-\mathrm{C})^{2} / \mathrm{m}^{6}\right]$

$C_{T} \quad=$ liquid-phase total TOC concentration

[mol- $\left.\mathrm{C} / \mathrm{m}^{3}\right]$

$=$ effective surface diffusion coefficient $\quad\left[\mathrm{cm}^{2} / \mathrm{s}\right]$

$k_{s} a=$ volumetric coefficient of intraparticle mass transfer

$Q \quad=$ ultimate uptake capacity of adsorbent

$q \quad=$ probability density of solid-phase TOC

concentration $\left[(\mathrm{mol}-\mathrm{C})^{2} / \mathrm{kg} \cdot \mathrm{m}^{3}\right]$

$R \quad=$ radius of adsorbent particle $\quad[\mathrm{cm}]$

$t=$ time [s]

$x \quad=$ Langmuir coefficient $\quad\left[\mathrm{m}^{3} / \mathrm{mol}-\mathrm{C}\right]$

$\beta_{n} \quad=$ parameter in Eq. (6) $\quad\left[(\mathrm{mol}-\mathrm{C})^{n} / \mathrm{s} \cdot \mathrm{m}^{3 n}\right]$

〈Superscripts〉

$n \quad=$ order of the term in Eq. (6)

$* \quad=$ at equilibrium

\section{Literature Cited}

1) Glückauf, E.: Trans. Faraday Soc., 51, 1540 (1955).

2) Komiyama, H. and J. M. Smith: AIChE J., 20, 728 (1974).

3) Miura, K., H. Kurahashi, Y. Inokuchi and K. Hashimoto J. Chem. Eng. Japan, 12, 281 (1979).

4) Okazaki, M., H. Kage, F. Iijima and R. Toei: J. Chem. Eng. Japan, 14, 26 (1981).

5) Suzuki, M. and K. Kawazoe: Seisankenkyu, 26, 275 (1974).

6) idem: J. Chem. Eng. Japan, 8, 379 (1975). 